\title{
Enhancement of gene transfer efficiency in the Bcap-37 cell line by dimethyl sulphoxide and menthol
}

\author{
JIAN LIN, LI QI ZHU, TAO QIN, QING HUA YU and QIAN YANG \\ Key Laboratory of Animal Physiology and Biochemistry, Ministry of Agriculture, \\ Nanjing Agricultural University, Nanjing, Jiangsu 210095, P.R. China
}

Received February 2, 2012; Accepted May 18, 2012

DOI: $10.3892 / \mathrm{mmr} .2012 .1084$

\begin{abstract}
Simple and efficient gene transfer into the nucleus would facilitate non-viral gene delivery. One promising method of non-viral gene delivery is to apply penetration enhancers. Chemicals, such as dimethyl sulfoxide (DMSO) and menthol, may have promise as non-toxic vehicles in improving gene transfer efficiency. In this study, the cytotoxic effects of DMSO and menthol were evaluated using MTT assays. Gene delivery efficiency in a human breast cancer cell line (Bcap-37) was investigated by quantitative PCR, fluorescence microscopy and flow cytometry. Non-toxic concentrations of DMSO (2\%) and menthol $(12.5 \mu \mathrm{M})$ enhanced the efficiency of liposomemediated gene delivery in Bcap-37 cells. Quantitative PCR results showed that growth hormone $(\mathrm{GH})$ mRNA expression in the post-menthol and pre-DMSO treatment groups was 10 -fold higher compared to that in the liposome group, while in the pre-menthol and post-DMSO treatment groups, a 30-fold increase in GH mRNA expression was observed. Both DMSO and menthol treatments increased green fluorescent protein (GFP) expression efficiency as shown by fluorescence microscopy experiments. Compared to the liposome group, the number of positive cells in the pre-menthol and postDMSO treatment groups was significantly increased by $15 \%$. Furthermore, cell cycle analysis demonstrated that there were significant differences among the DMSO-treated group, the menthol-treated group and the normal group, which implied different effects of DMSO and menthol treatments. In conclusion, both non-toxic and harmless DMSO (2\%) and menthol $(12.5 \mu \mathrm{M})$ treatments improve gene transfer efficiency, while post-DMSO treatment may be the most effective protocol in increasing transgene expression efficiency.
\end{abstract}

Correspondence to: Professor Qian Yang, Key Laboratory of Animal Physiology and Biochemistry, Ministry of Agriculture, Nanjing Agricultural University, Weigang 1, Nanjing, Jiangsu 210095, P.R. China

E-mail: yangqian11023@126.com

Key words: dimethyl sulfoxide, menthol, gene delivery, transfection efficiency, cell cycle

\section{Introduction}

One of the most important steps that limits transfection efficiency in non-viral gene delivery is the entry of nucleic acids across various membrane barriers and eventually into the nucleus where transcription occurs $(1,2)$. Therefore, studies have focused on increasing transfection efficiencies by passing through the nuclear pore (3). Yet, despite significant diversification of gene delivery strategies, efforts to expand the chemicals amenable to DNA-mediated gene transfer continue to stumble over a recurrent obstacle, the nuclear pore. Different methods have been used to deliver plasmid DNA across nuclear membranes.

An alternative approach to improve transfection efficiency is to incorporate penetration enhancers in formulations (4). New and effective transfection enhancers (non-ionic surfactants, bile salts and menthol) have been used to increase gene transfer efficiency (5). Another encouraging method to increase transfection efficiency is to disturb the cell cycle which significantly affects transfection efficiency. Cells treated with dimethyl sulfoxide (DMSO) disturb the hydrophobic function of the nuclear pore $(6,7)$. Brunner et al demonstrated that the addition of DMSO affects the transfer of plasmid DNA by influencing the expression of genes related to the cell cycle and cell hydrophobic properties (8).

DMSO has been commonly used for a number of years, not only for laboratory, but also for clinical purposes. In recent studies, DMSO was used as an efficient penetration enhancer for gene transfer expression (9-11). Li et al (12) revealed that the transfection of exogenous DNA incubated with DMSO was more efficient than without any treatment.

It has been found that menthol acts as a penetration enhancer by passing through the membrane $(13,14)$. Menthol has been extensively used in many aspects of pharmaceutical preparation. However, to the best of our knowledge, no report has described menthol as a penetration enhancer for the gene delivery system (4).

The exact conditions of how the chemicals, DMSO and menthol, successfully enhance gene transfer efficiency remain unclear. Unless plasmids enter the nucleus, they cannot be transcribed (15). Our study presents a novel approach that allows the expression of plasmid DNA in the Bcap-37 human breast cancer cell line after treatment with DMSO and menthol for the purposes of transgene expression. We aimed to explore 
the ability of DMSO to deliver genes into the nucleus. We also examined the ability of menthol to enhance the permeation of plasmid DNA. The aim of our study was to evaluate the safety and efficacy of using DMSO and menthol as permeability enhancers in gene delivery systems.

\section{Materials and methods}

Plasmid DNA and cell line. Plasmid DNA pAC-GFP-N1 was kindly provided by the College of Life Sciences at the Nanjing Agricultural University (Nanjing, China). Plasmid DNA pGN was kept in our laboratory. Information regarding the vector map and basic description is shown in Fig. 1. Plasmid DNA was amplified using Escherichia coli DH5a and purified using the E.Z.N.A.A ${ }^{\circledast}$ Endo-Free Plasmid Mini kit I (Omega, Norcross, GA, USA).

The Bcap-37 human breast cancer cell line (ER', p53 mutated) was purchased from the Shanghai Cell Collection, CAS (Shanghai, China). Bcap-37 cells were cultured in DMEM (Gibco) medium, supplemented with $10 \%$ fetal bovine serum (Gibco) at $37^{\circ} \mathrm{C}$ in a humidified atmosphere of $5 \% \mathrm{CO}_{2}$.

Succinimidyl-[4-(psoralen-8-yloxy)]-butyrate (SPB) was purchased from Pierce. Peptide derivative SPB-NLS was synthesized by Sangon Biotech (Shanghai, China) with the following sequences: SPB-PKKKRKV.

In vitro cytotoxicity assays (MTT assays). The cytotoxic effects of DMSO (J\&K) and menthol (J\&K) on the Bcap-37 cell line were evaluated by MTT assays. Approximately 5,000 Bcap-37 cells per population were plated in a flat-bottomed 96-well plate and incubated at $37^{\circ} \mathrm{C}$ for $24 \mathrm{~h}$ before the assays. Preliminary studies were performed to define the optimal concentration of DMSO and menthol which was suitable for testing. DMSO, ranging from 0.5 to $10 \%(\mathrm{v} / \mathrm{v})$, was added into the Bcap-37 cells to evaluate the toxicity. After incubation with DMSO for $48 \mathrm{~h}$ at $37^{\circ} \mathrm{C}$ in complete medium, the medium was removed and the cells were rinsed with PBS 3 times, then $75 \mu \mathrm{l}$ complete medium and $25 \mu \mathrm{l}$ MTT $(2 \mathrm{mg} / \mathrm{ml}$ in PBS) were added for another $4 \mathrm{~h}$. After $4 \mathrm{~h}$, the medium was aspirated and replaced with $100 \mu \mathrm{l}$ DMSO to dissolve formazan crystals. Absorbance was measured at $540 \mathrm{~nm}$, with untreated cells serving as the control. The same experiments were carried out to define the safe working concentration of menthol. The concentration of menthol that was added into the cells to evaluate the toxicity ranged from 12.5 to $400 \mu \mathrm{M}$.

Quantitative RT-PCR studies of transfection efficiency. Bcap-37 cells were incubated at $37^{\circ} \mathrm{C}$ for $48 \mathrm{~h}$ before transfection. Transfection was performed with the pGN plasmid (Lipofectamine ${ }^{\circledR} 2000$ Reagent; Invitrogen ${ }^{\mathrm{TM}}$ ) following the instructions of the manufacturer. The primers used for detecting growth hormone $(\mathrm{GH})$ mRNA expression are listed in Table I. The cells were divided into 12 groups (Tables II and III). Two groups were separately treated with $12.5 \mu \mathrm{M}$ menthol and 2\% DMSO $48 \mathrm{~h}$ before transfection. SPB-NLS/ DNA complexes were prepared $30 \mathrm{~min}$ before transfection. Another 2 groups were separately treated with $2 \%$ DMSO and $12.5 \mu \mathrm{M}$ menthol $2 \mathrm{~h}$ after transfection. DMEM medium was replaced with complete medium $6 \mathrm{~h}$ after transfection. GH mRNA expression was analyzed by quantitative RT-PCR
Vectors map and basic description of vectors

\begin{tabular}{llc}
\hline Plasmid name & \multicolumn{1}{c}{ Function } & Size (kb) \\
\hline pGN & express growth hormone & 23.8 \\
pAC-GFP-N1 & express GFP protein & 4.7 \\
\hline
\end{tabular}
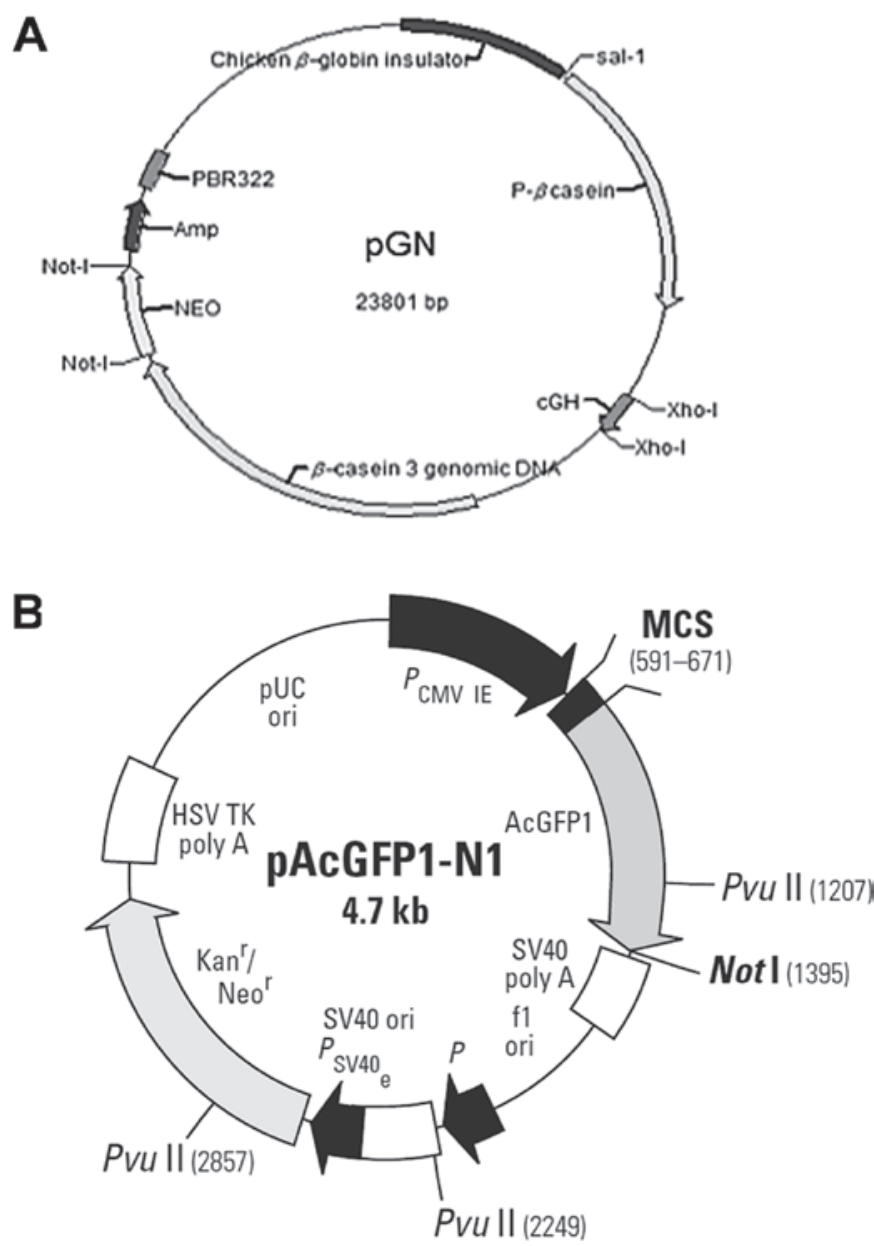

Figure 1. Vector map and basic description of vectors. (A) The pGN plasmid was constructed by our laboratory for the specific expression of growth hormone in the mammary gland to increase milk production. The pGN plasmid was constructed under the bone plasmid, $\mathrm{pBC1}$, which was a 21.6-kb vector designed to facilitate the expression of recombinant proteins in the milk of transgenic animals. Successful expression of recombinant protein in transgenic mice has generally been indicative of successful expression in larger animals, such as goats or cows. The pGN plasmid was a $23.8-\mathrm{kb}$ vector which was difficult to be transfected in producing transgenic animals. We searched for a transfection enhancing agent and the pGN plasmid was used to evaluate the transfection efficiency. (B) pAcGFP1-N1 encodes green fluorescent protein (GFP) from Aequorea coerulescens (excitation maximum, $475 \mathrm{~nm}$; emission maximum, $505 \mathrm{~nm}$ ). The coding sequence of the AcGFP1 gene contains silent base changes, which correspond to human codon-usage preferences. The MCS in pAcGFP1-N1 is between the immediate early promoter of CMV (PCMV IE) and the AcGFP1 coding sequences. Genes cloned into the multiple cloning site (MCS) will be expressed as fusions to the N-terminus of AcGFP1 if they are in the same reading frame as AcGFP1, and there are no intervening stop codons. SV40 polyadenylation signals downstream of the AcGFP1 gene direct proper processing of the $3^{\prime}$ end of the AcGFP1 mRNA. The vector backbone also contains an SV40 origin for replication in mammalian cells expressing the SV40 T antigen. A neomycin-resistance cassette (Neo'), consisting of the SV40 early promoter, the neomycin/kanamycin resistance gene of Tn5 and polyadenylation signals from the herpes simplex virus thymidine kinase (HSV TK) gene, allows stably transfected eukaryotic cells to be selected using G418. A bacterial promoter upstream of the gene expresses kanamycin resistance in Escherichia coli (E. coli). The pAcGFP1-N1 backbone also provides a pUC origin of replication for propagation in E. coli and an $\mathrm{f} 1$ origin for single-stranded DNA production. 
Table I. Quantitative RT-PCR primers used in detecting the pGN plasmid.

\begin{tabular}{llll}
\hline Gene & Sense primer & Anti-sense primer & Product length (bp) \\
\hline GH & gagaagctgaaggacctgga & tacgtctccgtcttgtgcag & 194 \\
Bcap-37 $\beta$-actin & gatcattgctcctcctgagc & tgtggacttgggagaggact & 385 \\
\hline
\end{tabular}

Table II. Relative GH mRNA expression in the DMSO-treated groups.

\begin{tabular}{|c|c|c|c|c|}
\hline Treatment & Blank & Control (liposome) & Liposome/pGN & Liposome/SPB-NLS/pGN \\
\hline Untreated & $1.039 \pm 0.349$ & $2.182 \pm 0.329$ & $11.006 \pm 1.909$ & $47.648 \pm 4.620$ \\
\hline Pre-DMSO treatment & & & $97.087 \pm 11.091$ & $100.521 \pm 10.032$ \\
\hline Post-DMSO treatment & & & $344.605 \pm 50.708$ & $155.090 \pm 12.675$ \\
\hline
\end{tabular}

Table III. Relative GH mRNA expression in the menthol-treated groups.

\begin{tabular}{lcccc}
\hline Treatment & Blank & Control (liposome) & Liposome/pGN & Liposome/SPB-NLS/pGN \\
\hline Untreated & $1.031 \pm 0.31$ & $3.132 \pm 1.676$ & $9.749 \pm 1.608$ & $70.677 \pm 18.145$ \\
Pre-menthol treatment & & & $277.110 \pm 23.371$ & $2,256.667 \pm 178.046$ \\
Post-menthol treatment & & $98.183 \pm 10.666$ & $30.560 \pm 3.057$ \\
\hline
\end{tabular}

$48 \mathrm{~h}$ after transfection. All transfection experiments were performed in triplicate.

Fluorescence microscopy studies of transfection efficiency. After detecting the mRNA expression levels, we evaluated protein expression efficiency in the DMSO- and mentholtreated groups. The pAC-GFP-N1 plasmid was used to evaluate the transgene expression efficiency. Bcap-37 cells were seeded at $10,000 /$ well in a flat-bottomed 12 -well plate $24 \mathrm{~h}$ prior to the experiments. Transfection was performed as mentioned above. After transfection, the cells were incubated for $48 \mathrm{~h}$ at $37^{\circ} \mathrm{C}$ for green fluorescent protein (GFP) expression. After incubation, the cells were washed twice with phosphate-buffered saline (PBS). Images were obtained by standard fluorescence microscopy (Olympus).

Flow cytometry analysis of transfection efficiency. As the results of fluorescence microscopy showed improved GFP expression efficiency in the DMSO- and menthol-treated groups, we aimed to calculate the increased percentage of transfected cells in the different groups. The capacity of flow cytometry for the rapid, individual analysis of a large number of cells make it ideally adapted for the study of transfection efficiency. Transfection was performed as mentioned above with the pAC-GFP-N1 plasmid. Cells were harvested and washed 3 times by PBS $48 \mathrm{~h}$ after transfection. Finally, the harvested cells were suspended in $500 \mu \mathrm{l}$ cold PBS and examined by a FACSCalibur flow cytometer, which was equipped with an argon laser (488 $\mathrm{nm})$.

Flow cytometry analysis of cell cycle. Previous studies have suggested that DMSO and menthol treatments significantly increase gene delivery efficiency $(14,16)$. In this study, in order to determine the mechanism by which the chemicals, DMSO and menthol, improve gene transfer efficiency, we examined the cell cycle changes in the different groups by flow cytometry. Bcap-37 cells were treated with DMSO and menthol $48 \mathrm{~h}$ before the assays. The cells were harvested and washed 3 times with PBS. The cells were then treated with RNase A $(5 \mu \mathrm{g} / \mathrm{ml})$ for $10 \mathrm{~min}$ at room temperature, followed by staining with propidium iodide (PI; $5 \mu \mathrm{g} / \mathrm{ml}$ ), a DNA-binding dye for $2 \mathrm{~h}$ at room temperature. Subsequently, the cells were subjected to flow cytometry to analyze the cell cycle changes.

Statistical analysis. Statistical analyses of transfection efficiency and cell cycle changes were carried out using one-way analysis of variance (ANOVA). A P-value $<0.05$ denoted statistically significant differences. All data are expressed as the means \pm standard error of the mean.

\section{Results}

In vitro cell viability. One of the major requirements for gene delivery is low cytotoxicity. In order to define the safe working concentration of DMSO and menthol for the Bcap-37 cells, we performed MTT assays. The viability of Bcap-37 cells in the absence or presence of various concentrations of DMSO is shown in Fig. 2A. The results showed that cell viability was surpassed by $>90 \%$ when the concentration of DMSO was $<1 \%(\mathrm{v} / \mathrm{v})$. However, cell viability only exceeded $80 \%$ when the concentration of DMSO was $<2 \%(\mathrm{v} / \mathrm{v})$, and significantly decreased at higher concentrations of DMSO [2.5 and $5 \%(\mathrm{v} / \mathrm{v})]$. The minimum cell viability of Bcap-37 cells was 17.2 in $10 \%$ DMSO. As shown in Fig. 2A, among 8 tested 


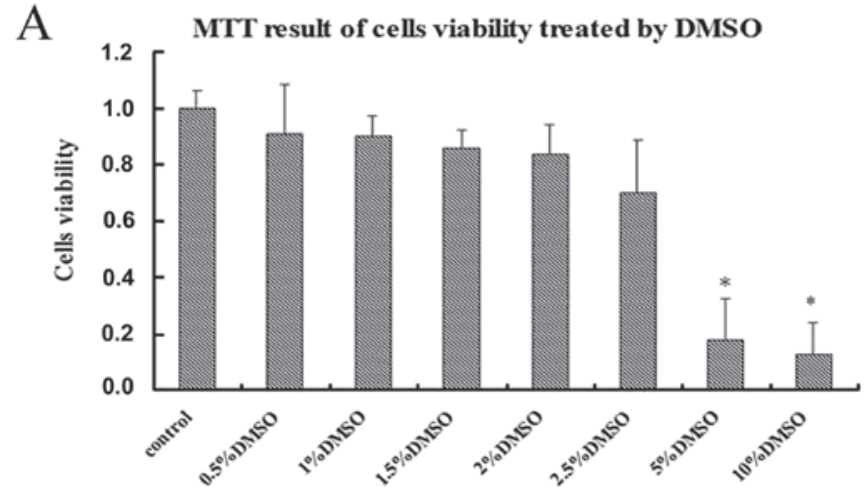

B

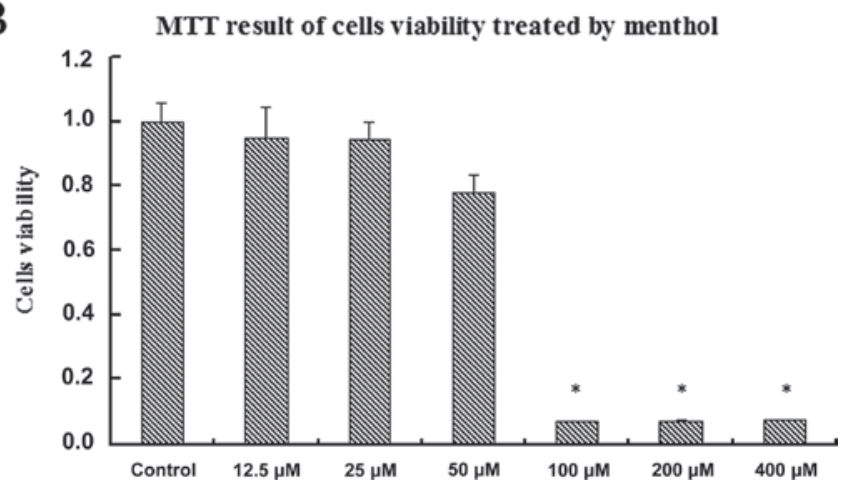

Figure 2. Cell viability of DMSO and menthol treatments. (A) MTT results of cell viability treated by DMSO. (B) MTT results of cell viability treated by menthol.

concentrations of DMSO, $2 \%(\mathrm{v} / \mathrm{v})$ DMSO was the optimum working concentration.

We also detected the working concentration of menthol. The viabilities in the absence or presence of various concentrations of menthol are shown in Fig. 2B. We observed a strange phenomenon: cell viability was surpassed by $>80 \%$ when the concentration of menthol was $<50 \mu \mathrm{M}$. Once the concentration of menthol was up to $100 \mu \mathrm{M}$, cell viability sharply decreased to $<10 \%$ (Fig. 2B). According to the literature, cell proliferation speed significantly decreases when cells are treated with high concentrations of menthol (17). According to the afore-mentioned results, we selected 2\% DMSO and $12.5 \mu \mathrm{M}$ menthol for our experiments.

\section{Transfection efficiency experiments}

DMSO and menthol enhance GH mRNA expression in $S P B-N L S$-meditated transfection. We hypothesized that a correlation existed between the ability of penetration enhancers to wreck membranes under natural conditions and penetrating efficiency. To analyze the transfection efficiency of DMSO and menthol treatments, we carried out transient transfection with the pGN plasmid in the Bcap-37 cell line.

The results showed that the expression of GH mRNA in the pre-DMSO treatment group was 10 -fold higher than that in the liposome/pGN group. Compared to the liposome/pGN group, the post-DMSO treatment group showed a significantly enhanced expression of GH mRNA (up to 30-fold). However, the pre-DMSO/SPB-NLS co-treatment group showed no difference in GH mRNA expression levels compared to the pre-DMSO treatment group (both groups showed a 10 -fold
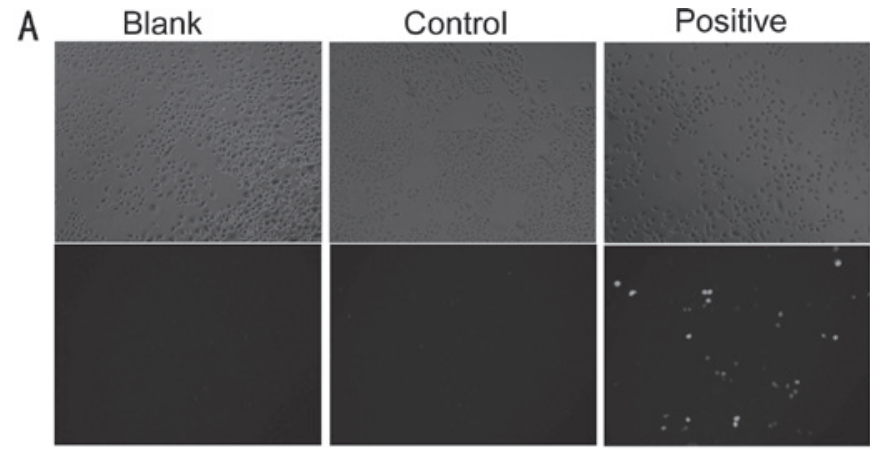

B
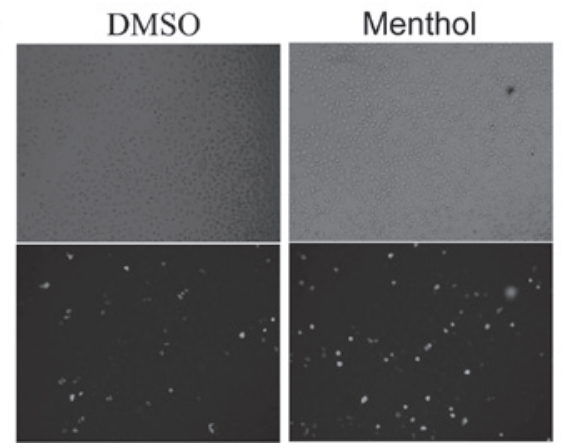

Figure 3. Fluorescence microscopy results of transfection efficiency (A) Fluorescence microscopy results of blank, control and liposome groups (B) Fluorescence microscopy results of the DMSO- and menthol-treated groups.

increase compared to the liposome/pGN group), while the post-DMSO/SPB-NLS co-treatment group showed even lower levels in GH mRNA expression compared to the post-DMSO treatment group (Table II).

Similar results were also observed in the menthol-treated groups. Compared to the liposome/pGN group, the expression of GH mRNA was greatly increased in the post-menthol treatment group (10-fold), and significantly enhanced in the pre-menthol treatment group (30-fold). However, there were 2 differences compared to the DMSO-treated groups. One was that the pre-menthol/SPB-NLS co-treatment group had significantly increased GH mRNA expression levels (up to 200 -fold compared to the liposome/pGN group). The other was that menthol treatment only showed high transcriptional activities in the pre-menthol treatment group and the prementhol/SPB-NLS co-treatment group (Table III).

Fluorescence microscopy of transfection efficiency. After detecting GH mRNA expression, we aimed to evaluate the function of DMSO and menthol in improving protein expression efficiency by fluorescence microscopy. Protein expression efficiency was evaluated using the pAC-GFP-N1 plasmid, which expressed GFP as a reporter protein. Normal cells were used as the blank group. Cells transfected with the pGN plasmid were used as the negative control group. The results showed that there was no GFP expression in the blank and negative groups, while only small areas of fluorescence were observed in the liposome/pAC-GFP-N1 group (Fig. 3A). Compared to the liposome/pAC-GFP-N1 group, the expression of GFP in the DMSO- and menthol-treated groups was much more efficient (Fig. 3B). 
Table IV. Cell cycle analysis of the different treatments.

\begin{tabular}{lcccc}
\hline Treatment & G1 phase $(\%)$ & S phase $(\%)$ & G2 phase $(\%)$ & Apoptosis \\
\hline Blank & $61.41 \pm 0.42$ & $31.58 \pm 0.89$ & $7.01 \pm 0.47$ & $1.11 \pm 0.29$ \\
Liposome/pGN & $56.79 \pm 0.87$ & $38.93 \pm 2.30$ & $4.29 \pm 1.43$ & $2.21 \pm 0.59$ \\
DMSO & $71.41 \pm 1.33$ & $19.45 \pm 1.14$ & $9.14 \pm 0.24$ & $1.40 \pm 0.10$ \\
Menthol & $57.48 \pm 0.79$ & $34.31 \pm 1.45$ & $8.21 \pm 0.76$ & $0.22 \pm 0.05$ \\
\hline
\end{tabular}

Flow cytometry analysis of transfection efficiency. Our main goal was to develop a convenient and efficient delivery system for functional gene transfer. As the fluorescence microscopy results demonstrated a high GFP expression efficiency in the DMSO- and menthol-treated groups, we aimed to calculate the increased percentage of transfected cells in the different groups by flow cytometry. The flow cytometry results showed that the positive percentages of the blank, control (liposome) and negative groups (liposome/pGN) were $0.91,1.32$ and $0.87 \%$, respectively. Compared to the $8.55 \%$ positive percentage in the liposome/pAC-GFP-N1 group, the positive percentage in the pre-DMSO treatment group (9.58\%) and the post-DMSO treatment group (10.91\%) was significantly increased. However, the positive percentages in the pre-menthol treatment group (7.9\%) and the post-menthol treatment group (7.44\%) were slightly lower compared to the liposome/pAC-GFP-N1 group (8.55\%). These results were consistent with those obtained by fluorescence microscopy. Data analysis also implied that the highest transfection efficiency was observed in the SPB-NLS/postDMSO co-treatment group (15.81\%), which was significantly higher than that in the SPB-NLS/pre-menthol co treatment group (12.97\%) (Fig. 4).

Cell cycle analysis. Although high efficiency was demonstrated in gene transfer, the mechanism by which DMSO and menthol improve gene delivery remained unclear. Cell cycle synchronization was confirmed to have a crucial role in gene delivery. The flow cytometry results showed that there were significant differences in the cell cycle between the blank group (G1 phase, 61.41\%; G2 phase, 7.01\%; S phase, $31.58 \%$ ) and the liposome group (G1 phase, $56.79 \%$; G2 phase, $4.29 \%$; S phase, $38.93 \%$ ). All 3 phases of the cell cycle were affected by liposome treatment (Table IV). Data analysis showed that the cell cycle phases were G1 57.48\%, G2 8.21\% and $\mathrm{S} 34.31 \%$ in the menthol-treated group, and $\mathrm{G} 171.41 \%$, G2 $9.14 \%$ and S $19.45 \%$ in the DMSO-treated group, which were significantly different from the blank group. DMSO and menthol exposure resulted in an alteration in the percentage of nuclei, mainly in the $\mathrm{S}$ and G1 phases. A significant increase in the percentage of nuclei in the $S$ phase and a significant decrease in the percentage of nuclei in the G1 phase occurred following DMSO treatment. Menthol treatment only led to a significant increase in the percentage of nuclei in the G1 phase and a significant decrease in the percentage of nuclei in the $S$ phase. It should also be noted that the significant changes in the percentage of nuclei in the G1 and S phases was observed at the working concentration of menthol and DMSO, without altering the G2 phase. Previous studies using synchronized cells showed that cells expressed between 50- and 3,000-fold more gene product when transfected in the G2 or G2-M phase as compared to those transfected in the G1 phase $(8,18)$.

\section{Discussion}

Previously, methods of in vitro transfection of different cells were limited to electric transfection (19), liposome-mediated(4) or retroviral infection (20). In this study, our main goal was to develop a convenient and efficient delivery system for functional gene delivery. Our results prove that the addition of DMSO and menthol enhances liposome-mediated transfection efficiency in Bcap-37 cells. Furthermore, we found that transfection efficiency in the post-DMSO treatment group (21) had improved compared to that in the pre-DMSO treatment group, while the efficiency of gene transfer in the pre-menthol treatment group was extremely higher compared to that in the post-menthol treatment group. In addition, we confirmed that gene delivery efficiency in the DMSO/SPB-NLS and menthol/ SPB-NLS groups had greatly improved compared to that in the DMSO- or menthol-treated groups. Although peptide derivative SPB-NLS has been shown to be an effective transfection reagent in vitro (data not shown), it has never been used in conjunction with DMSO or menthol. DMSO and menthol have been shown to improve the transfection efficiency of plasmid DNA by increasing the permeability of cell membranes (22), the integrity of the nucleus (23) or by affecting cell cycle synchronization $(24,25)$.

Quantitative evaluation of gene transfer. Quantitative RT-PCR was conducted to evaluate the transfection efficiency of the different treatments (DMSO and menthol). The results revealed that the expression of GH mRNA in the DMSO- and menthol-treated groups was significantly higher than that in the liposome-mediated group. This result was consistent with the study by Jain and Gewirtz (16). Our results clearly demonstrated that the post-DMSO treatment group expressed much more GH mRNA than the pre-DMSO treatment group, which suggests, as also shown by a previous study, that DMSO affects the nuclear pore in gene transfer (23). Taken together, our results show that post-DMSO treatment is most effective in enhancing gene delivery. Of note, the DMSO/SPB-NLS co-treatment was less efficient in enhancing gene transfer compared to the post-DMSO treatment group. Although the exact mechanism of the DMSO-mediated gene delivery remains unknown, our results clearly demonstrate that the addition of DMSO influences the transfection efficiency. We speculated, as also shown in a previous study, that pre-DMSO treatment may increase transfection efficiency by regulating the gene controlled cell cycle and cell hydrophobic properties (26), while post-DMSO 
$\mathrm{E}$

A

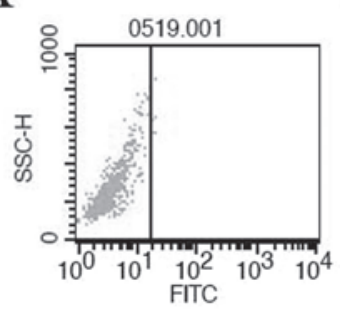

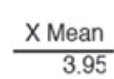

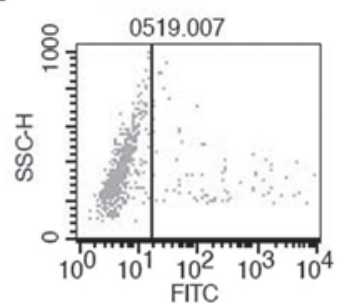

B
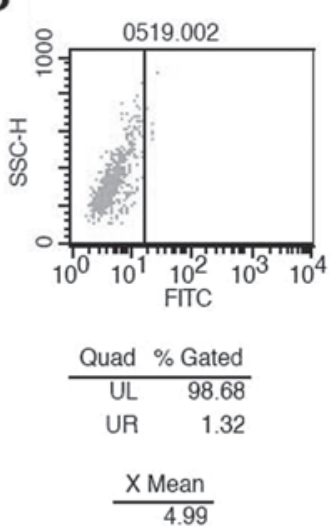

$\mathrm{F}$
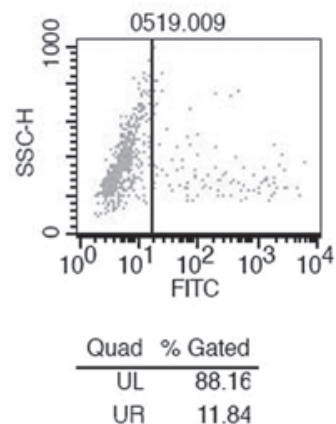

$\frac{\mathrm{X} \text { Mean }}{91.54}$

$\mathrm{J}$

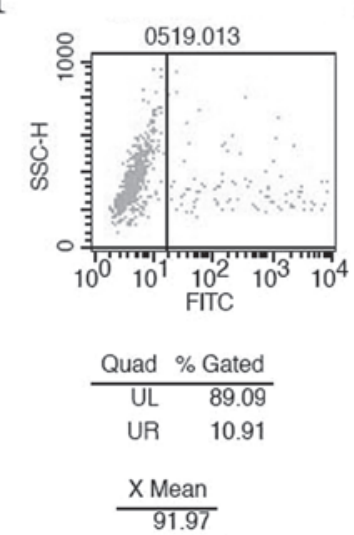

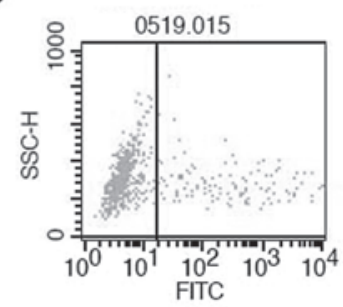

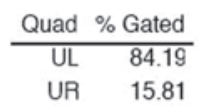

$\frac{\mathrm{X} \text { Mean }}{133.00}$
$\mathrm{C}$
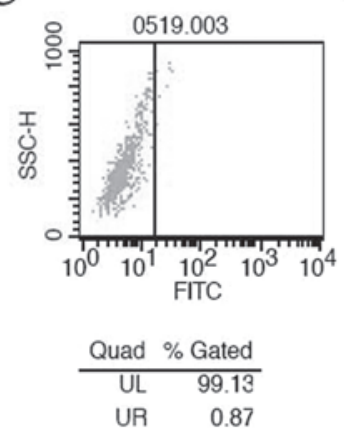

$\frac{X \text { Mean }}{5.00}$

G
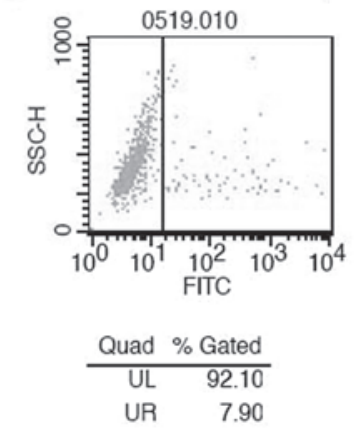

$\frac{X \text { Mean }}{65.80}$

$\mathrm{K}$
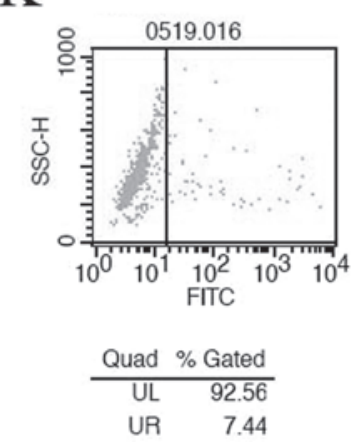

$\frac{X \text { Mean }}{69.55}$
$\mathrm{D}$
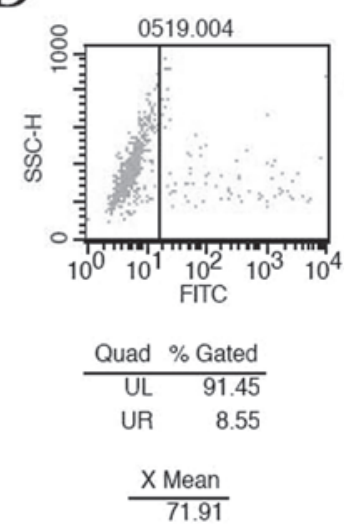

$\mathrm{H}$
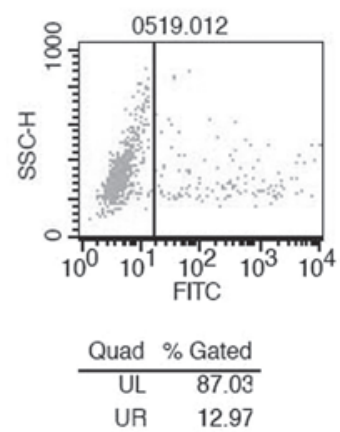

$\frac{X \text { Mean }}{135.67}$

$\mathrm{L}$
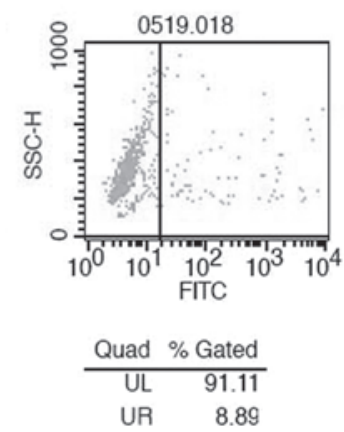

$\frac{X \text { Mean }}{88.22}$

Figure 4. Flow cytometry results of transfection efficiency. (A) Blank group; (B) liposome group (control group); (C) liposome/pGN group (negative group); (D) liposome/pAC-GFP-N1 group (positive group); (E) post-menthol treatment group; (F) post-menthol/SPB-NLS co-treatment group; (G) pre-menthol treatment group; (H) pre-menthol/SPB-NLS co-treatment group; (I) post-DMSO treatment group; (J) post-DMSO/SPB-NLS co-treatment group; (K) pre-DMSO treatment group; (L) pre-DMSO/SPB-NLS co-treatment group.

treatment may improve transfection efficiency by influencing the formation of the nuclear pore.

Similar results were also found in the menthol-treated groups. What was different from the DMSO-treated groups was that pre-menthol treatment had more significant results compared to post-menthol treatment concerning GH mRNA expression. Another difference was that the menthol/SPB-NLS co-treatment group achieved supreme transfer efficiency. As for the reason why menthol/SPB-NLS co-treatment had such an impact on transfection efficiency, it remains to be further investigated.
Quantitative estimation of gene expression. After we investigated the function of DMSO and menthol in improving $\mathrm{GH}$ mRNA expression, we evaluated protein (GFP) expression efficiency in the DMSO- and menthol-treated groups by fluorescence microscopy and flow cytometry. Flow cytometry measurements made every accurate determination of fluorescence intensities feasible. The results from both analyses showed that DMSO and menthol treatments increased GFP expression efficiency. DMSO and menthol treatments only slightly improved the expression of GFP. These results are completely 
different from those obtained by qRT-PCR. The reason behind this phenomenon may be the following: the slight improvement in GFP expression in the DMSO- and menthol-treated groups implied that DMSO and menthol treatments may affect cell viability, which in turn limits gene expression.

We also found that the post-DMSO/SPB-NLS co-treatment group achieved the highest GFP expression efficiency (increased by $90 \%$; flow cytometry). Similarly, the pre-menthol/SPB-NLS co-treatment group also obtained a high efficiency in GFP expression (increased by 52\%; flow cytometry). Notably, highlevel transcription efficiency (DMSO- and menthol-treated group) did not always mean high-level expression efficiency. Among all treatments, the post-DMSO/SPB-NLS and the prementhol/SPB-NLS co-treatment groups not only showed high transcription levels (GH mRNA expression levels), but also displayed high translation levels (GFP expression levels).

Methods involved in improving gene transfer efficiency. Apart from some isolated reports $(27,28)$, the majority of studies have aimed at increasing DNA nuclear import by adding NLS-containing proteins or using novel polymers. There has been relatively little success transforming information gathered into more effective methods for DNA delivery in vitro. We aimed to search for versatile solutions to improve transfection efficiency. DMSO and menthol seemed to be a suitable way to solve this problem. Previous studies using DMSO have shown that this amphiphilic molecule affects transfection by influencing the formation of the nuclear pore (6). DMSO also alters the cell cycle to accomodate gene transfer. Cell cycle synchronization has been confirmed to be a crucial part in gene delivery $(29,30)$. Previous studies using synchronized cells have shown that cells express more gene product when transfected in the G2 or G2-M phase $(8,18)$. DNA microinjected into the nucleus produces robust gene expression, while cytoplasm injection results in low expression levels. Thus, if plasmids were presented in the cytoplasm, they could enter into the nucleus when the envelope was disrupted (30). It was well known that non-dividing or growth-arrested cells cannot be easily transfected. By contrast, cells undergoing mitosis are much more receptive to transfection (31). The results from the present study demonstrate that DMSO stops the cell cycle during mitosis, which explains the high gene delivery efficiency in the DMSO-treated groups.

Menthol is a naturally-occurring cyclic terpene alcohol of plant origin, which has been frequently used since antiquity for medicinal purposes. However, as a penetration enhancer, the ability of menthol in improving gene delivery efficiency has not been reported. Menthol may be used to increase the permeability of cell membranes or to affect the integrity of the nucleus (23). As detected in previous experiments, the mechanism by which menthol enhances transmembrane permeation may include the possible reversible disruption of the intercellular lipid domain $(20,21)$.

Although high transfection efficiency has been demonstrated, issues related to the effects of DMSO or menthol on transfection efficiency and the mechanisms have not been fully addressed. The present investigation aimed to generate versatile solutions to further improve transfection efficiency. It would be hopeful to provide impetus for searching for more versatile solutions in transferring plasmid DNA.
In conclusion, DMSO can increase the transfection efficiency by influencing the pore integrity of the nucleus or regulating the gene controlled cell cycle and cell hydrophobic properties. Additionally, it is likely that menthol facilitates the gene transfer efficiency by increasing the permeability of cell membranes. Our results clearly demonstrate that pre-menthol and postDMSO treatments both improve gene delivery efficiency.

The pre-menthol and post-DMSO treatments are developing techniques. We stronlgy believe that only versatile transfection techniques would aid in the development of a controlled release gene transfer method, unlike many of the conventional nucleic acid carriers that are purely liposome-based. Hopefully, our research will lead to a safer alternative to viral systems for gene delivery.

\section{Acknowledgements}

This study was supported by grants from the National Animal Transgenic Breeding Grand Project (nos. 2008ZX08008-004 and 2011ZX08008-004).

\section{References}

1. Lechardeur D and Lukacs GL: Intracellular barriers to non-viral gene transfer. Curr Gene Ther 2: 183-194, 2002.

2. Leong KW, Mao HQ, Truong-Le VL, Roy K, Walsh SM and August JT: DNA-polycation nanospheres as non-viral gene delivery vehicles. J Control Release 53: 183-193, 1998.

3. Kamiya H, Tsuchiya H, Yamazaki J and Harashima $H$ : Intracellular trafficking and transgene expression of viral and non-viral gene vectors. Adv Drug Deliv Rev 52: 153-164, 2001.

4. Zhou M, Liu H, Xu X, et al: Identification of nuclear localization signal that governs nuclear import of BRD7 and its essential roles in inhibiting cell cycle progression. J Cell Biochem 98: 920-930, 2006.

5. Man N, Yu L, Zheng F, Li Y and Wen LP: Efficient gene transfer to rat fetal osteoblastic cells by synthetic peptide vector system. Protein Pept Lett 16: 368-372, 2009.

6. Notman R, den Otter WK, Noro MG, Briels WJ and Anwar J: The permeability enhancing mechanism of DMSO in ceramide bilayers simulated by molecular dynamics. Biophys J 93: 2056-2068, 2007.

7. Ribbeck K and Gorlich D: The permeability barrier of nuclear pore complexes appears to operate via hydrophobic exclusion. Embo J 21: 2664-2671, 2002.

8. Brunner S, Sauer T, Carotta S, Cotten M, Saltik M and Wagner E: Cell cycle dependence of gene transfer by lipoplex, polyplex and recombinant adenovirus. Gene Ther 7: 401-407, 2000.

9. Guillard EC, Laugel C and Baillet-Guffroy A: Molecular interactions of penetration enhancers within ceramides organization: a FTIR approach. Eur J Pharm Sci 36: 192-199, 2009.

10. Hewitt PG,Poblete N, Wester RC, Maibach HI and Shainhouse JZ: In vitro cutaneous disposition of a topical diclofenac lotion in human skin: effect of a multi-dose regimen. Pharm Res 15: 988-992, 1998.

11. Mittal A, Sara UV, Ali A and Aqil M: The effect of penetration enhancers on permeation kinetics of nitrendipine in two different skin models. Biol Pharm Bull 31: 1766-1772, 2008.

12. Li L, Shen W, Min L, Dong H, Sun Y and Pan Q: Human lactoferrin transgenic rabbits produced efficiently using dimethylsulfoxide-sperm-mediated gene transfer. Reprod Fertil Dev 18: 689-695, 2006.

13. Brain KR, Green DM, Dykes PJ, Marks R and Bola TS: The role of menthol in skin penetration from topical formulations of ibuprofen 5\% in vivo. Skin Pharmacol Physiol 19: 17-21, 2006.

14. Ho HO, Chen LC, Lin HM and Sheu MT: Penetration enhancement by menthol combined with a solubilization effect in a mixed solvent system. J Control Release 51: 301-311, 1998.

15. Bremner KH, Seymour LW, Logan A and Read ML: Factors influencing the ability of nuclear localization sequence peptides to enhance nonviral gene delivery. Bioconjug Chem 15: 152-161, 2004. 
16. Jain PT and Gewirtz DA: Enhancement of liposomal gene delivery in human breast cancer cells by dimethyl sulfoxide. Int J Mol Med 1: 609-611, 1998.

17. Fiore M, Zanier R and Degrassi F: Reversible G(1) arrest by dimethyl sulfoxide as a new method to synchronize Chinese hamster cells. Mutagenesis 17: 419-424, 2002.

18. Escriou V, Carriere M, Bussone F, Wils P and Scherman D: Critical assessment of the nuclear import of plasmid during cationic lipid-mediated gene transfer. J Gene Med 3: 179-187, 2001.

19. Mir LM: Nucleic acids electrotransfer-based gene therapy (electrogenetherapy): past, current, and future. Mol Biotechnol 43: 167-176, 2009.

20. Ramezani A and Hawley RG: Strategies to insulate lentiviral vector-expressed transgenes. Methods Mol Biol 614: 77-100, 2010.

21. Heckert RA, Elankumaran S, Oshop GL and Vakharia VN: A novel transcutaneous plasmid-dimethylsulfoxide delivery technique for avian nucleic acid immunization. Vet Immunol Immunopathol 89: 67-81, 2002.

22. Sultana Y, Jain R, Aqil M and Ali A: Review of ocular drug delivery. Curr Drug Deliv 3: 207-217, 2006.

23. Wang H, Zhong CY, Wu JF, Huang YB and Liu CB: Enhancement of TAT cell membrane penetration efficiency by dimethyl sulphoxide. J Control Release 143: 64-70, 2010.
24. Campeau P, Chapdelaine P, Seigneurin-Venin S, Massie B and Tremblay JP: Transfection of large plasmids in primary human myoblasts. Gene Ther 8: 1387-1394, 2001.

25. Migita S, Hanagata N, Tsuya D, Yamazaki T, Sugimoto Y and Ikoma T: Transfection efficiency for size-separated cells synchronized in cell cycle by microfluidic device. Biomed Microdevices 13: 725-729, 2011.

26. Srinivas S, Sironmani TA and Shanmugam G: Dimethyl sulfoxide inhibits the expression of early growth-response genes and arrests fibroblasts at quiescence. Exp Cell Res 196: 279-286, 1991.

27. Collas P and Alestrom P: Rapid targeting of plasmid DNA to zebrafish embryo nuclei by the nuclear localization signal of SV40 T antigen. Mol Mar Biol Biotechnol 6: 48-58, 1997.

28. Arenal A, Pimentel R, Garcia C, Pimentel E and Alestrom P: The SV40 T antigen nuclear localization sequence enhances nuclear import of vector DNA in embryos of a crustacean (Litopenaeus schmitti). Gene 337: 71-77, 2004

29. Medina-Kauwe LK, Xie J and Hamm-Alvarez S: Intracellular trafficking of nonviral vectors. Gene Ther 12: 1734-1751, 2005.

30. Xavier J, Singh S, Dean DA, Rao NM and Gopal V: Designed multi-domain protein as a carrier of nucleic acids into cells. J Control Release 133: 154-160, 2009.

31. Dean DA, Strong DD and Zimmer WE: Nuclear entry of nonviral vectors. Gene Ther 12: 881-890, 2005. 